\title{
Estimación de rasgos funcionales en dos especies arbóreas de una gradiente altitudinal tropical en el Centro de Perú
}

\author{
Estimation of functional traits in two tree species of \\ a tropical elevational gradient in central Peru
}

Humberto Zelada ${ }^{1, *}$ y Carlos Reynel ${ }^{2}$

Recibido: 10 marzo 2018 | Aceptado: 06 octubre 2019 | Publicado en línea: 15 diciembre 2019 Citación: Zelada, H; Reynel, C. 2019. Estimación de rasgos funcionales en dos especies arbóreas de una gradiente altitudinal tropical en el Centro de Perú. Revista Forestal del Perú 34(2): 132-143. DOI: http://dx.doi.org/10.21704/rfp.v34i2.1323

\begin{abstract}
Resumen
En el actual contexto mundial de cambio climático, estudiar los rasgos funcionales de los árboles resulta de gran importancia, ya que contribuye al conocimiento de la capacidad de adaptación de éstos a las variaciones impuestas por el ambiente, debido a la estrecha relación que tienen sus rasgos con diversos procesos agronómicos, biológicos, ambientales y fisiológicos. En este sentido, y teniendo en cuenta que esta temática en el Perú ha sido escasamente abordada; en el presente trabajo se reporta un análisis de los resultados preliminares sobre la estimación de tres rasgos funcionales en dos especies arbóreas registradas en una gradiente altitudinal de la Reserva Comunal El Sira. Los rasgos funcionales estimados fueron: área foliar, área foliar específica y densidad básica de la madera; encontrándose que los atributos de estos rasgos mostraron variación según el nivel altitudinal. Los hallazgos de este estudio representan una primera aproximación para tratar cuestiones ecológicas relacionadas con rasgos funcionales de árboles en gradientes altitudinales del Perú.
\end{abstract}

Palabras clave: ecología vegetal, cambio climático, Neea, Theobroma, zonas protegidas

\footnotetext{
${ }^{1}$ Consultor Independiente.

${ }^{2}$ Herbario Forestal MOL, Facultad de Ciencias Forestales, Universidad Nacional Agraria La Molina (UNALM), Av. La Molina s/n, La Molina, Lima, Perú.

* Autor de Correspondencia: humbertzg07@gmail.com
} 


\begin{abstract}
In the current global context of climate change, to study the functional traits of trees are very important, as it contributes to know the relationship between its adapting and variations imposed by various agronomic, biological, environmental and physiological processes. In this sense, and considering that this aspect has scarcely been treated in Peru, the present work reports an analysis of preliminary results on the estimation of three functional traits in two tree species, which are located, in one elevational gradient of El Sira Communal Reserve. The functional traits analyzed were: leaf area, specific leaf area and basic wood density; founding that the attributes of these traits showed variation according to the elevational level. The results of this study represent a first approach to deal ecological issues related to functional traits of trees along elevational gradients of Peru.
\end{abstract}

Palabras clave: plant ecology, climate change, Neea, Theobroma, protected areas

\section{Introducción}

La importancia de estudiar los rasgos funcionales en ecosistemas tropicales de montaña, radica principalmente en poder analizar las respuestas de los organismos a las variaciones impuestas por el ambiente y explicar ecológicamente la relación de una planta con su entorno biótico y abiótico (Violle et al. 2007), en ambientes donde existe una alta diversidad vegetal que se relaciona con una gran diversidad de arquitecturas, tamaños y morfologías (Andrade 2005).

En este contexto, los gradientes altitudinales tropicales constituyen laboratorios naturales para estudiar el comportamiento de las especies vegetales y su adaptación al cambio climático (Valenzuela et al. 2015).

Violle et al. (2007) indican que los rasgos funcionales son características estructurales que modulan las plantas a fin de que su desarrollo no se vea restringido; siendo las hojas y el tallo donde suelen expresarse mayormente estas modulaciones (Jiménez-Noriega 2014). De esta manera, los rasgos de hojas habitualmente estudiados son: área foliar, área foliar específica, contenido de materia seca, fuerza tensil, contenido de nitrógeno y contenido de fósforo; en tanto que para los rasgos del tallo son: densidad básica de madera y altura máxima del árbol (Violle et al. 2007).

Asimismo, el área foliar se define como la superficie de las hojas de las plantas medidas en el haz o envés; mientras que el área foliar específica se refiere a la cantidad de área de hoja que existe por unidad de peso de la misma (Ruiz 2015). Ambos rasgos, usados como indicadores de adaptación ambiental, se relacionan con las estrategias de captación y absorción de luz y agua (Cardoza 2011). Por su parte, la densidad básica de la madera, se refiere a la relación que existe entre el peso seco de una sección leñosa de una planta -tallo, raíz, ramas, etc.- y el volumen de la misma sección cuando se encuentra saturado de agua (Álvarez et al. 2013), y expresa la respuesta a la perturbación del medio que se observa a través del crecimiento lento o rápido de las especies arbóreas (Bermeo 2010).

Bermeo (2010), calculó que valores promedio bajos de área foliar y de área foliar especifica $-326.14 \mathrm{~cm}^{2}$ y $129.80 \mathrm{~cm}^{2} / \mathrm{g}$, respectivamente- se dan en especies arbóreas tropicales conforme aumenta la altitud debido a la modulación de sus hojas al estar en climas más hostiles. Chave et al. (2006) mencionan que la densidad básica de la madera decrece con el aumento en altitud y presenta diferencias significativas con las regiones de baja altitud. Adicionalmente, Bermeo (2010) sugiere que estas diferencias se deben principalmente a las condiciones ambientales donde se desarrollan las especies arbóreas.

Ante ello, el objetivo del presente trabajo es contribuir al conocimiento de la ecología de especies arbóreas dando cuenta de los resultados preliminares en la evaluación de tres rasgos 
funcionales: área foliar, área foliar específica y densidad básica de la madera, en dos especies arbóreas localizadas en una gradiente altitudinal de la Reserva Comunal El Sira (RCS), para tener una primera aproximación de como varía el valor, promedio, de estos rasgos en un ambiente cambiante. Asimismo, se proponen algunas recomendaciones para investigaciones futuras.

\section{Materiales y Métodos}

\section{Área de estudio}

El área de estudio se encuentra en el distrito de Yuyapichis, provincia de Puerto Inca, departamento de Huánuco; en la zona centro oriental del Perú y, en una gradiente altitudinal entre los 250 a $2250 \mathrm{msnm}$.

El estudio se realizó en cinco parcelas permanentes de 1 ha distribuidas en una gradiente altitudinal de la RCS. Cuatro de las parcelas se encontraban dentro de la RCS y una en el Área de Conservación Privada Panguana (ACPP). El ACPP es un área protegida que se encuentra en la zona de amortiguamiento de la RCS, a 30 minutos en bote de la ciudad de Yuyapichis. Las parcelas permanentes están situadas en tres zonas de vida: (1) bosque húmedo - Tropical (bh-T); (2) bosque húmedo - Tropical (bh-T) transicional a bosque muy húmedo - Premontano Tropical (bmh-PT) y; (3) bosque pluvial-Premontano Tropical (bp-PT) (INRENA 1995; Valenzuela et al. 2015; SENAMHI 2017) (Cuadro 1 y Figura 1).

\section{Colección de muestras}

La metodología empleada en el presente estudio fue una adaptación siguiendo las recomendaciones del manual de campo propuesto por Patiño (2005); y del protocolo elaborado por el Proyecto Madidi et al. (2012).

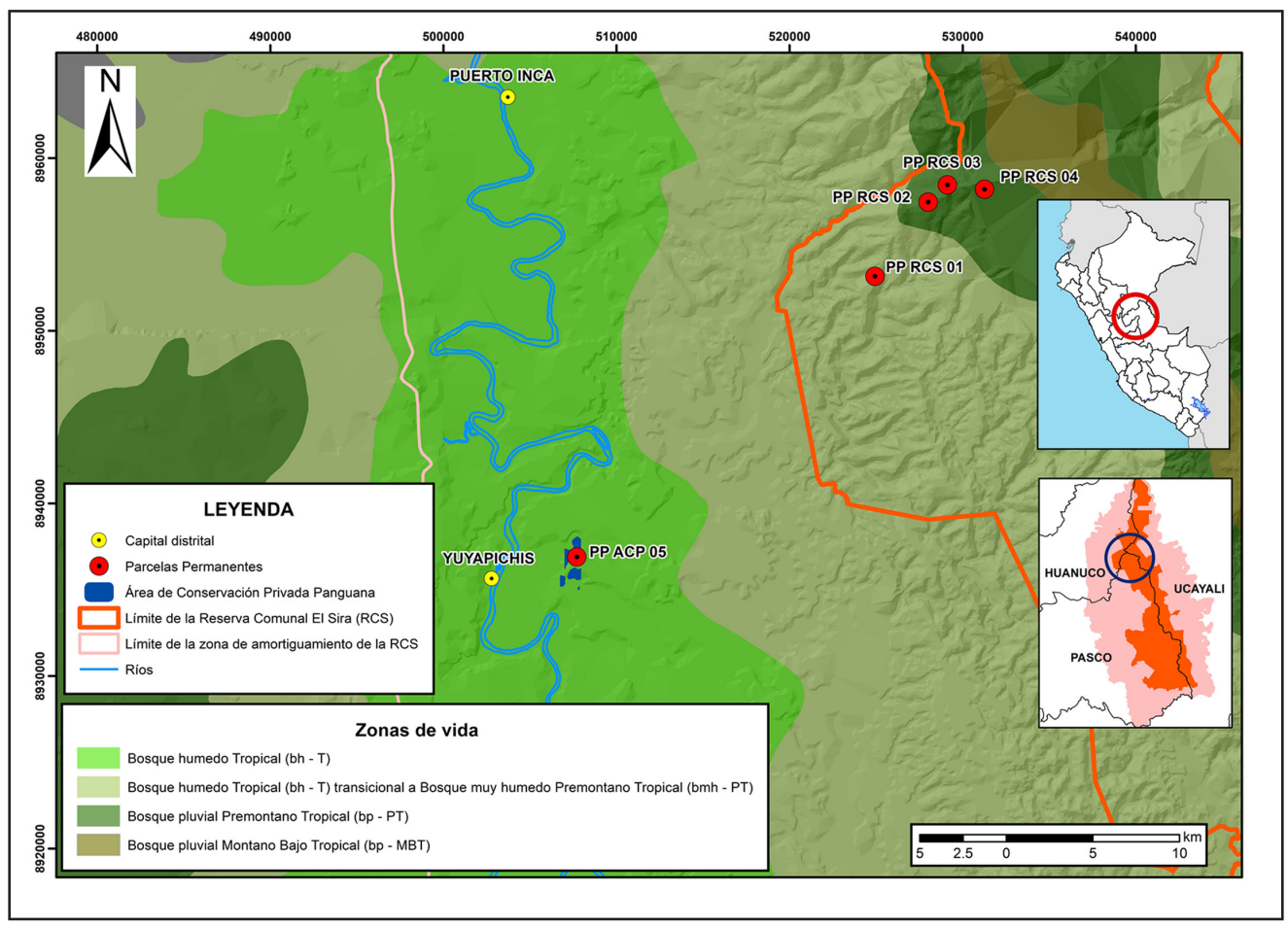

Figura 1. Mapa del área de estudio según las zonas de vida. 


\begin{tabular}{|c|c|c|c|c|c|c|}
\hline \multirow{3}{*}{ 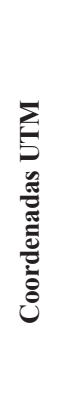 } & II & 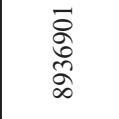 & $\frac{\tilde{n}}{\tilde{n}}$ & 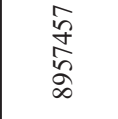 & $\begin{array}{l}\tilde{w} \\
⿱ 亠 䒑 \\
\infty \\
\infty \\
\infty\end{array}$ & 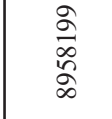 \\
\hline & $z$ & 莡 & 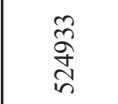 & $\begin{array}{l}\text { के } \\
\text { क्. } \\
\text { مn }\end{array}$ & $\frac{\tilde{a}}{\mathrm{~d}}$ & 息 \\
\hline & 气ूँ & $\stackrel{\infty}{\rightarrow}$ & $\stackrel{\infty}{\rightarrow}$ & $\stackrel{\infty}{\rightarrow}$ & $\stackrel{\infty}{\rightarrow}$ & $\stackrel{\leftrightarrow}{\rightarrow}$ \\
\hline \multicolumn{2}{|c|}{ 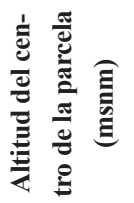 } & $\vec{n}$ & $\frac{q}{\infty}$ & $\overline{్ ర ి ~}$ & $\begin{array}{l}\infty \\
\stackrel{0}{\circ}\end{array}$ & స్తి \\
\hline \multicolumn{2}{|c|}{ 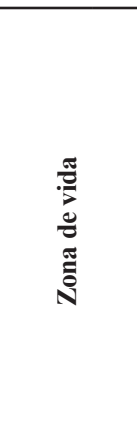 } & 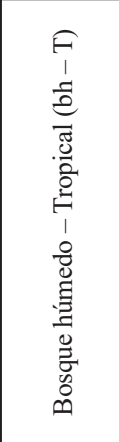 & 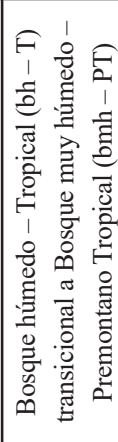 & \multicolumn{3}{|c|}{ 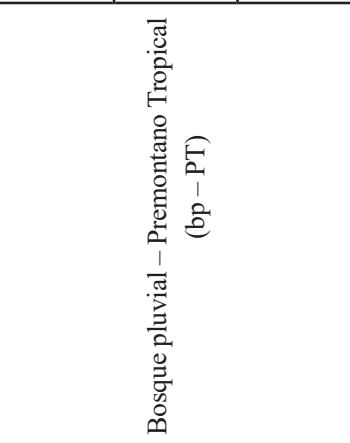 } \\
\hline \multicolumn{2}{|c|}{ 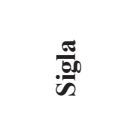 } & 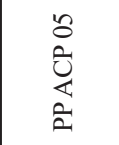 & 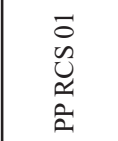 & 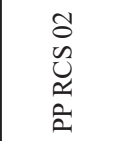 & 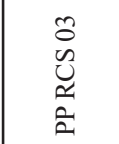 & 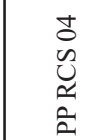 \\
\hline \multicolumn{2}{|c|}{$\frac{\pi}{\pi}$} & 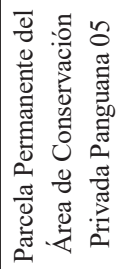 & 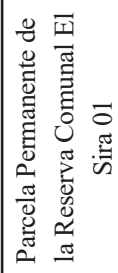 & 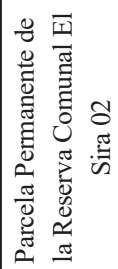 & 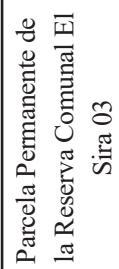 & 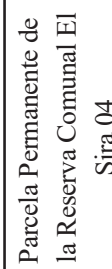 \\
\hline \multicolumn{2}{|c|}{$\stackrel{\circ}{Z}$} & - & $N$ & $m$ & + & in \\
\hline
\end{tabular}

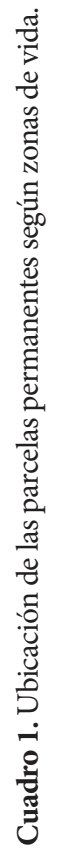


Además, al tratarse de un estudio con resultados preliminares, que priorizó la posibilidad de obtener muestras en diferentes niveles altitudinales, se optó por muestrear un número reducido de árboles por cada parcela, dado que realizar trabajos de investigación en bosques montanos húmedo representa grandes retos, por la lejanía, la inaccesibilidad y por su alta biodiversidad (Valenzuela et al. 2015).

Los criterios considerados para la selección de los árboles, fueron: a) que tengan un DAP mayor a $10 \mathrm{~cm}$; b) que sean lo suficientemente altos para que la copa esté expuesta a la luz solar y; c) fueron seleccionados como mínimo cinco y como máximo diez arboles dentro de cada parcela, indistintamente de la especie a la que pertenezcan.
Luego, de cada árbol se obtuvo una rama de la copa, se priorizó aquellas que estén más expuestas a la luz. Se seleccionaron y colectaron hojas presentes en la rama. En las hojas compuestas cada foliolo fue contabilizado (Figura 2). Para este estudio no se tomó en consideración la posición que ocupaba la rama respecto al eje vertical - basal, medial, apical - u horizontal - norte, sur, este, oeste- del árbol.

\section{Estimación del área foliar}

Para la estimación del área foliar $\left(\mathrm{cm}^{2}\right)$ se siguieron las recomendaciones de Patiño (2005). La representatividad de las hojas seleccionadas dependió del daño causado por herbívoros o patógenos, así como del tamaño, color y forma promedio. De ese modo, la can-

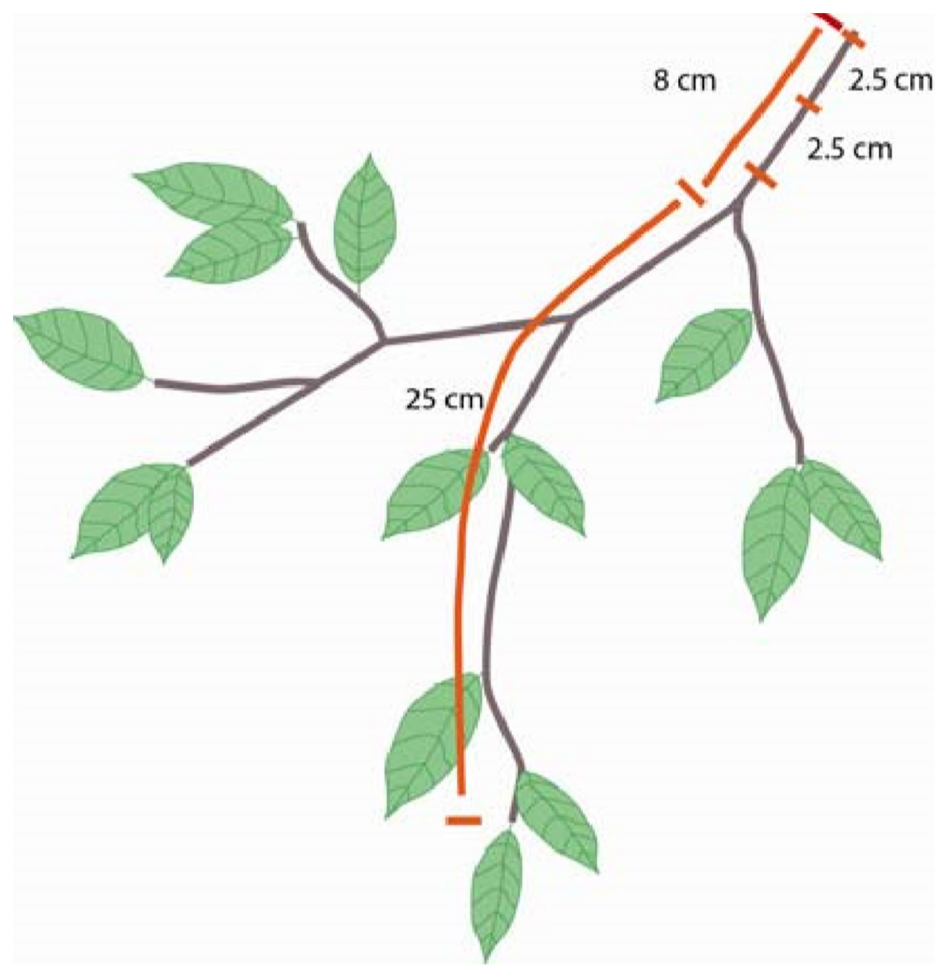

Figura 2. Esquema de la colecta de rama. 


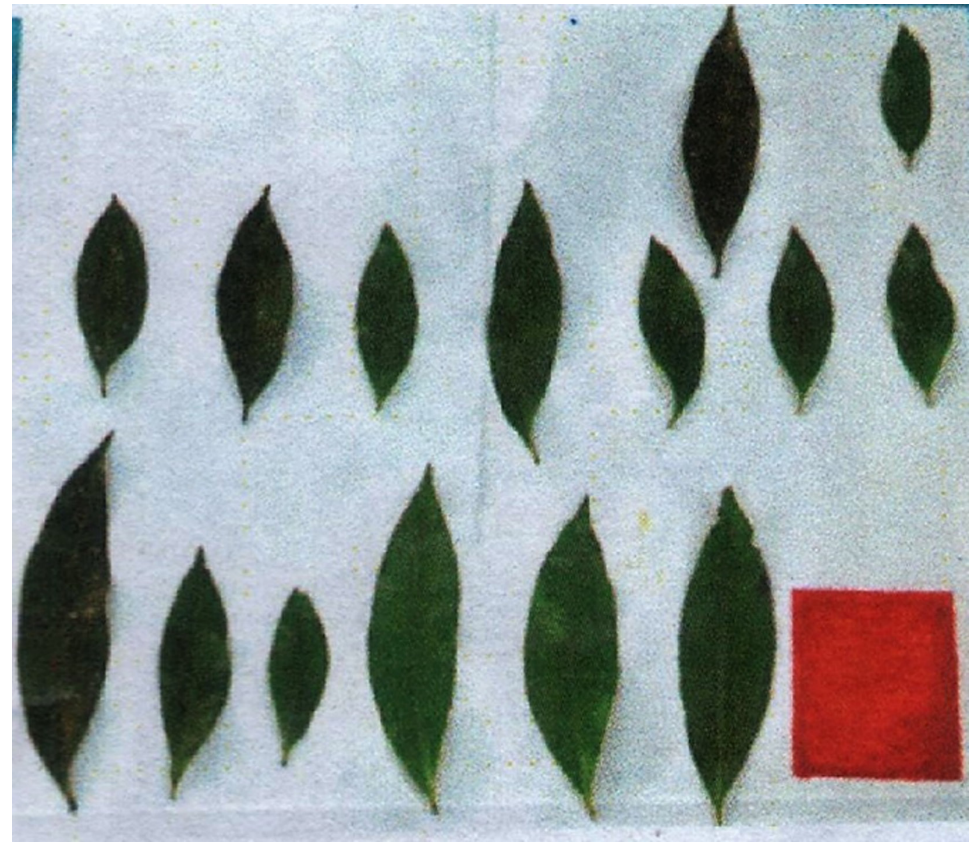

Figura 3. Modelo de hojas seleccionadas para la estimación del área foliar.

tidad de hojas por cada árbol, de las diferentes especies colectadas, varió entre 10 a 20 .

Posteriormente, esta cantidad de hojas representativa, se colocó en una cartulina A2 (43×28 $\mathrm{cm}, 300 \mathrm{~g})$ donde se dibujó previamente un cuadrado de $10 \times 10 \mathrm{~cm}$ pintado de color: magenta, azul o rojo. Las hojas se colocaron con la misma dirección, es decir, con el ápice apuntando hacia la parte superior de la cartulina y lo más estirada que se pudieran, evitando siempre poder dañarlas. Luego, a estas hojas colocadas en la cartulina A2, se les tomaron fotografías con una cámara digital bajo el mismo ángulo (Figura 3) y con el uso del programa de análisis estadístico R, se determinó el área foliar. Se procuró tomar las fotografías lo más pronto posible para evitar la sequedad de las hojas.

\section{Estimación del área foliar especifica}

Se calculó el peso seco en estufa - por tres días a $70^{\circ} \mathrm{C}$ - para luego aplicar la relación recomendada por Pérez et al. (2004): área foliar especifica $=$ área foliar $\left(\mathrm{cm}^{2}\right) /$ peso seco $(\mathrm{g})$.

\section{Estimación de la densidad básica de la made-} ra

Empleando una tijera de mano, se extrajeron dos segmentos, de cada rama colectada, por cada árbol. La longitud de estos segmentos fue de $10 \mathrm{~cm}$ cada uno (Figura 4).

Con estos segmentos, se estimó la densidad básica a través de la fórmula: densidad básica de la madera = peso seco $(\mathrm{g}) /$ volumen verde $\left(\mathrm{cm}^{3}\right)$; donde, el peso seco se obtuvo colocando los segmentos en estufa - por 76 horas a $100^{\circ} \mathrm{C}$ - y el volumen verde empleando el método conocido como dimensional o de Arquímedes (Figura 5). Luego, se promedió aritméticamente los valores de cada segmento obteniendo un solo valor por árbol.

Es importante señalar que el cálculo de la densidad básica se realizó considerando la corteza de la rama, ya que, en muchos estudios se ha demostrado que el peso de la corteza puede representar una proporción importante del volumen y peso total de la rama (Baraloto et 


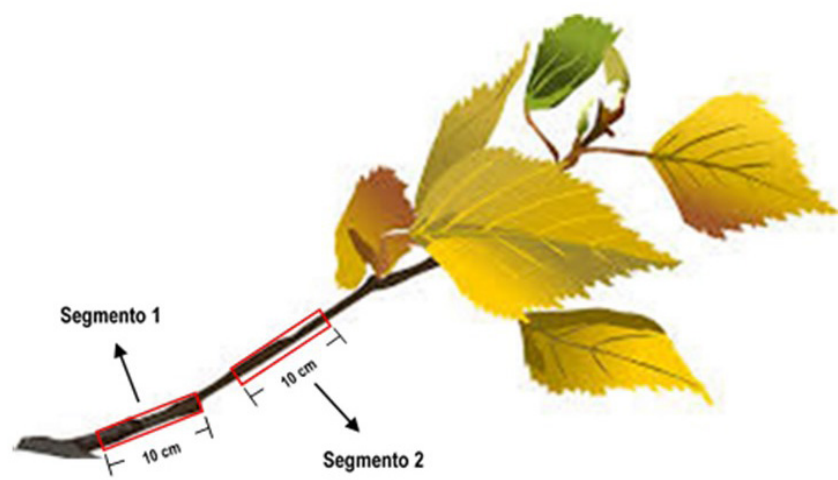

Figura 4. Modelo de los segmentos de rama colectados para la estimación de la densidad básica.

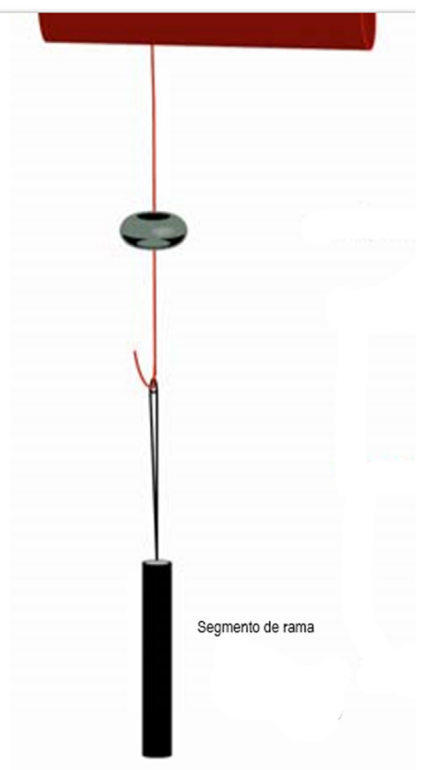

al. 2010; Sarmiento et al. 2011; Álvarez et al. 2013).

\section{Análisis de datos}

Después del procesamiento de datos en campo y en gabinete, se calculó el valor promedio para cada uno de los rasgos funcionales evaluados, en los individuos muestreados utilizando el programa Microsoft Excel 2013. Asimismo, se realizó una comparación descriptiva de los valores promedio encontrados, según zona de vida o nivel altitudinal. Si bien durante la colecta de muestra se evaluaron diferentes especies (ver Cuadro 2), en los resultados del presente trabajo solo se mostrarán los correspondientes a las especies Theobroma subincanum Mart. (Malvaceae) y Neea divaricata Poepp. \& Endl. (Nyctaginaceae) por registrar individuos en diferentes zonas de vida, lo que permitió realizar una comparación altitudinal de los valores encontrados.

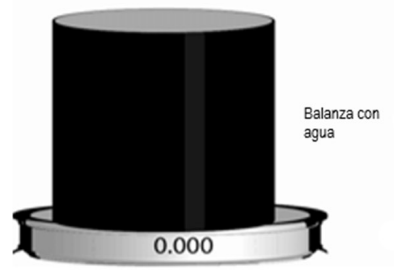

Figura 5. Esquema de medición del volumen verde de los segmentos de la rama.

\section{Resultados}

\section{Valor promedio del área foliar}

Theobroma subincanum: en la muestra evaluada del árbol de la zona de vida bh - T (350 - $650 \mathrm{msnm})$, se encontró un valor de $524.98 \mathrm{~cm}^{2}$ mientras que, para la muestra colectada del árbol de la zona transicional bh - T / bmh - PT (650 - 1050 msnm) se registró un valor de $1083.62 \mathrm{~cm}^{2}$. 


\begin{tabular}{|c|c|c|c|c|c|c|}
\hline \multirow{3}{*}{ Nombre científico } & \multicolumn{5}{|c|}{ Zona de vida } & \multirow{3}{*}{$\begin{array}{l}\text { Total de } \\
\text { árboles }\end{array}$} \\
\hline & \multirow{2}{*}{$\begin{array}{c}\text { bh - T } \\
\text { PP ACP } \\
05 \\
\end{array}$} & \multirow{2}{*}{$\begin{array}{c}\text { bh - T } \\
\text { / bmh - } \\
\text { PT } \\
\text { PP RCS } \\
01 \\
\end{array}$} & \multicolumn{3}{|c|}{ bp - PT } & \\
\hline & & & $\begin{array}{c}\text { PP RCS } \\
02\end{array}$ & $\begin{array}{c}\text { PP RCS } \\
03\end{array}$ & $\begin{array}{c}\text { PP RCS } \\
04\end{array}$ & \\
\hline Neea divaricata Poepp. \& Endl. & 0 & 1 & 2 & 0 & 0 & 3 \\
\hline Dendrobangia boliviana Rusby & 0 & 0 & 2 & 0 & 0 & 2 \\
\hline Ladenbergia macrocarpa (Vahl) Klotzsch & 0 & 0 & 0 & 0 & 2 & 2 \\
\hline Psychotria sp. & 0 & 0 & 2 & 0 & 0 & 2 \\
\hline Schefflera patula (Rusby) Harms & 0 & 0 & 0 & 0 & 2 & 2 \\
\hline Theobroma subincanum Mart. & 1 & 1 & 0 & 0 & 0 & 2 \\
\hline Weinmannia cochensis Hieron. & 0 & 0 & 0 & 0 & 2 & 2 \\
\hline Aspidosperma parvifolium A. DC. & 1 & 0 & 0 & 0 & 0 & 1 \\
\hline Castilla ulei Warb. & 1 & 0 & 0 & 0 & 0 & 1 \\
\hline Celtis schippii Standl. & 1 & 0 & 0 & 0 & 0 & 1 \\
\hline Cinchona hirsuta Ruiz \& Pav. aff. & 0 & 0 & 0 & 0 & 1 & 1 \\
\hline Clethra castaneifolia Meisn. & 0 & 0 & 0 & 0 & 1 & 1 \\
\hline Diploon cuspidatum (Hoehne) Cronquist & 1 & 0 & 0 & 0 & 0 & 1 \\
\hline Elaeagia karstenii Standl. & 0 & 0 & 0 & 1 & 0 & 1 \\
\hline Endlicheria arunciflora (Meisn.) Mez & 0 & 0 & 0 & 1 & 0 & 1 \\
\hline Eschweilera coriacea (DC.) S.A. Mori & 0 & 1 & 0 & 0 & 0 & 1 \\
\hline Hieronyma duquei Cuatrec. & 0 & 0 & 0 & 1 & 0 & 1 \\
\hline Hirtella elongata Mart. \& Zucc. & 1 & 0 & 0 & 0 & 0 & 1 \\
\hline Inga cecropietorum Ducke & 0 & 0 & 0 & 1 & 0 & 1 \\
\hline Leonia glycycarpa var. glycyacarpa & 1 & 0 & 0 & 0 & 0 & 1 \\
\hline Licania heteromorpha var. heteromorpha & 0 & 0 & 1 & 0 & 0 & 1 \\
\hline Maquira coriacea (H. Karst.) C.C. Berg & 1 & 0 & 0 & 0 & 0 & 1 \\
\hline Meriania hexamera Sprague & 0 & 0 & 0 & 1 & 0 & 1 \\
\hline Miconia elongate Cogn. & 0 & 0 & 0 & 0 & 1 & 1 \\
\hline Pouteria vernicosa T.D. Penn. aff. & 0 & 0 & 1 & 0 & 0 & 1 \\
\hline Protium altsonii Sandwith & 0 & 1 & 0 & 0 & 0 & 1 \\
\hline Protium amazonicum (Cuatrec.) Daly & 0 & 1 & 0 & 0 & 0 & 1 \\
\hline Total de árboles & 8 & 5 & 8 & 5 & 9 & 35 \\
\hline
\end{tabular}

Cuadro 2. Cantidad de árboles evaluados en el gradiente altitudinal según especie y zona de vida. 
Neea divaricata: en la muestra estudiada del árbol evaluado en la zona transicional bh $-\mathrm{T}$ / bmh - PT (650 - $1050 \mathrm{msnm})$ el valor estimado del área foliar fue $515.81 \mathrm{~cm}^{2}$; y para la muestra del árbol ubicado en la zona bp PT (1 050 - $2200 \mathrm{msnm}$ ) el valor obtenido fue $538.20 \mathrm{~cm}^{2}$.

\section{Valor promedio del área foliar especifica}

Theobroma subincanum: para la muestra del árbol evaluado en la zona de vida bh - T (350 - $650 \mathrm{msnm}$ ), el valor de área foliar especifica registrado fue $33.52 \mathrm{~cm}^{2} / \mathrm{g}$; en cambio, para la muestra del árbol ubicado en la zona transicional bh - T / bmh - PT (650 - $1050 \mathrm{msnm})$ el valor promedio de área foliar especifica fue $72.82 \mathrm{~cm}^{2} / \mathrm{g}$.

Neea divaricata: en la muestra estudiada del árbol de la zona transicional bh - T / bmh - PT (650 - $1050 \mathrm{msnm})$ se encontró un valor promedio de área foliar especifica de $77.21 \mathrm{~cm}^{2} / \mathrm{g}$; mientras que para la muestra evaluada del árbol de la zona de vida bp - PT (1 050 - 2200 $\mathrm{msnm}$ ) el valor registrado fue $190.17 \mathrm{~cm}^{2} / \mathrm{g}$.

\section{Valor promedio de la densidad básica}

Theobroma subincanum: para las muestras del árbol evaluado en la zona de vida bh - T (350 - $650 \mathrm{msnm})$ el valor promedio de la densidad básica de la madera encontrado fue 0.44 $\mathrm{g} / \mathrm{cm}^{3}$; y para las muestras del árbol estudiado en la zona transicional bh $-\mathrm{T} / \mathrm{bmh}-\mathrm{PT}(650$ - $1050 \mathrm{msnm}$ ) el valor encontrado para este mismo rasgo fue $0.38 \mathrm{~g} / \mathrm{cm}^{3}$.

Neea divaricata: el valor promedio de densidad básica de la madera encontrado en las muestras del árbol de la zona transicional bh - T / bmh - PT (650 - $1050 \mathrm{msnm}$ ) fue 0.51 $\mathrm{g} / \mathrm{cm}^{3}$; en cambio, para las muestras del árbol estudiado en la zona de vida bp - PT (1 050 $2200 \mathrm{msnm}$ ) el valor obtenido para este rasgo funcional fue $0.30 \mathrm{~g} / \mathrm{cm}^{3}$.

\section{Discusión}

Los valores promedio de área foliar encontrados para las dos especies aumentan conforme aumenta la altitud, observándose entre los arboles de Theobroma subincanum, un incremento mayor que al observado en los individuos de Neea divaricata. Bermeo (2010) reportó valores promedio bajos de área foliar $\left(326.14 \mathrm{~cm}^{2}\right)$ en árboles de bosques tropicales secundarios localizados por encima de los 500 msnm hasta los $2900 \mathrm{msnm}$. Por su parte, Enríquez (2017) encontró en especies arbóreas de un bosque siempreverde montano bajo (entre los 1996 y los $2042 \mathrm{msnm}$ ), valores promedio elevados $\left(885 \mathrm{~cm}^{2}\right)$ de área foliar debido, principalmente, a la naturaleza compuesta de sus hojas. Al respecto, Garnica y Saldarriaga (2015) señalaron que la variación del área foliar en gradientes altitudinales tropicales, está directamente relacionado con la variación de los factores ambientales, la geología, altitud y latitud, así como de los factores alométricos propios de cada especie arbórea.

Los valores promedio de área foliar específica hallados en ambas especies analizadas, aumentan con el nivel altitudinal, siendo, este incremento, 40 por ciento mayor entre los árboles de Neea divaricata; lo cual no concuerda, nuevamente, con lo planteado por Bermeo (2010), quien reportó que en zonas de mayor nivel altitudinal se espera encontrar menores valores promedio de área foliar especifica producto de las modulaciones de las plantas a la hostilidad climática en estos sitios. Sin embargo, Garnica y Saldarriaga (2015) señalaron que a medida que se avanza en el gradiente altitudinal, tanto el valor promedio del área foliar como del área foliar específica, aumentan, lo cual coincide con los hallazgos reportados en este estudio.

Respecto a la densidad básica de la madera, los resultados muestran una disminución del valor promedio de la densidad básica de la madera conforme aumenta el nivel altitudinal; lo cual coincide con lo señalado por Chave et al. (2006). De igual forma, Tyree y Ewers (1991) mencionaron que en lugares donde hay mayor radiación solar - zonas de vida de mayor altitud - se esperaría encontrar menor densidad básica de la madera ya que, las especies arbóreas deberían tener un sistema de transporte de agua eficiente para mantener su tasa de respiración - y fotosíntesis - elevada. 
Es preciso resaltar lo señalado por Violle et al. (2007), quienes indican que no existe un único valor o atributo para un determinado rasgo de una especie, ya que se pueden registrar valores muy diferentes a lo largo de gradientes altitudinales, inclusive con poca diferencia altitudinal, por lo cual, para tener mayores consistencias al respecto, es necesario realizar más mediciones y en ciclos climáticos, sobre todo.

\section{Consideraciones Finales}

Los resultados encontrados sobre los valores promedios del área foliar, área foliar específica y de la densidad básica de la madera, para una misma especie arbórea, sugieren una variación según la zona de vida o el nivel altitudinal; debido, muy probablemente, a las diferentes condiciones ambientales. Siendo esta una primera aproximación, es necesario aumentar el tamaño de muestra por cada especie.

Por ende, a partir de este estudio, se sugiere mejorar la estandarización de la metodología empleada, principalmente en los siguientes aspectos: i) homogenizar el número de árboles, por especie, a muestrear según zona de vida o nivel altitudinal; ii) considerar la posición vertical y los puntos cardinales de la rama a colectar; iii) asegurar que la cantidad y calidad de hojas y ramas sea lo más representativa posible del árbol seleccionado - dos a tres ramas por árbol es recomendable -; iv) procurar analizar las muestras lo antes posible después de su colección, sobre todo para el registro fotográfico de las hojas - área foliar - y el registro del peso húmedo de las ramas - densidad básica -

Finalmente, tomando en consideración los hallazgos encontrados y las sugerencias planteadas para investigaciones futuras; el análisis del comportamiento de especies arbóreas localizadas en gradientes altitudinales tropicales mediante el enfoque de ecología funcional -basado en rasgos funcionales-, puede contribuir a simplificar la complejidad florística y entender las respuestas de la vegetación ante variables ambientales, y también, a responder algunas interrogantes que aparecieron en este estudio, como, por ejemplo: ¿Existirá alguna correlación entre rasgos funcionales -diferentes- dentro de una misma especie arbórea?

\section{Agradecimientos}

Los autores agradecemos a la Jefatura de la Reserva Comunal El Sira (SERNANP), al Proyecto Co-gestión Amazonia Perú-GIZ, y al Laboratorio de Propiedades Físico - Mecánicas de la madera de la UNALM, por la colaboración brindada en la ejecución del presente estudio.

\section{Bibliografía}

Andrade, JL. 2005. Fisiología Ecológica de Árboles Tropicales: Avances y Perspectivas. Revista Chapingo 11 (2): 83-91.

Álvarez, E; Benítez, D; Velásquez, C; Cogollo, A. 2013. Densidad básica del fuste de árboles del bosque seco en la costa Caribe de Colombia. Revista Intrópica 8:17-28.

Bermeo, D. 2010. Determinación y caracterización de tipos funcionales de plantas (TFPs) en bosques secundarios dentro de un gradiente altitudinal y su relación con variables bioclimáticas. Tesis Mag. Sc. Turrialba, CR, CATIE. $114 \mathrm{p}$.

Calvo, M; Silva-Pando, FJ; Rozados, MJ; Díaz, M; Rodríguez, P; Duo, I. 2005. El índice de área foliar (LAI) en masas de abedul (Betula celtiberica Rothm. et Vasc.) en Galicia. Sociedad Española de Ciencias Forestales 20:111-116.

Cardoza, FS. 2011. Diversidad y Composición Florística y funcional de los bosques del Parque Nacional Monte Cristo, El Salvador. Tesis Mag. Sc. Turrialba, CR, CATIE. 127 p.

Chave, J; Muller-Landau, HC; Baker, TR; Easdale, TS; Ter Steege, H; Webb, CO. 2006. Regional and phylogenetic variation of wood density across 2,456 neotropical tree species. Ecological Applications 16(6):2356-2367.

Enríquez, V. 2017. Grupos funcionales y su papel en la restauración de paisajes en la región sur del Ecuador. Tesis Ing. Med. Amb. Loja, EC, UNL. 79 p. 
Garnica, C; Saldarriaga, S. 2015. Diversidad funcional en un gradiente altitudinal del complejo de paramos Sumapaz - Cruz verde. Tesis Ing. For. Bogotá, CO, UD. 73 p.

INRENA (Instituto Nacional de Recursos Naturales, Perú). 1995. Mapa Ecológico del Perú: Guía Explicativa. Lima, Perú. 271 p.

Patiño, S. 2005. Manual de campo para el estudio de hojas y madera editado para establecer efectos de sequía. PAN-AMAZONIA Project ene. 2005:1-12.

Pérez A, JA; Garcia M, E; Enriquez Q, JF; Quero C, AR; Perez, J; Hernandez G, A. 2004. Análisis de crecimiento, area foliar específica y concentración de nitrógeno en hojas de pasto "mulato" (Brachiaria híbrido, cv.). Tec. Pecu. Méx. 42(3):447-458.

Proyecto Madidi (Proyecto Inventario Florístico de la Región Madidi, BO); Herbario Nacional de Bolivia; Missouri Botanical Garden; UAM (Universidad Autónoma de Madrid, ES). 2012. Protocolo para la medición de características funcionales. La Paz, Bolivia. 13 p.

Ruiz, J. 2015. Grupos funcionales de plantas con potencial para la restauración ecológica de manantiales de agua en la microcuenca de la Laguna de Pedro Palo y sus alrededores, Cundinamarca - Colombia. Tesis Mag. Sc. Bogota, Universidad Nacional de Colombia. 123 p.

Sarmiento, C; Patiño, S; Paine, J; Beauchene, J; Thibaut, A; Baraloto, C. 2011. Withinindividual variation of trunk and branch xylem density in tropical trees. American Journal of Botany 98(1):140-149.

SENAMHI (Servicio Nacional de Metereologia e Hidrologia, Perú). 2017. Atlas de zonas de vida del Perú - Guia Explictiva. Eds. Aybar-Camacho, C; Lavado-Casimiro, W; Sabino, E; Ramírez, S; Huerta, J \& Felipe-Obando. Lima, Perú. 30 p.

Tyree, M; Ewers, T. 1991. The Hydraulic architecture of trees and other woody plants. Review New Phytol. 119(34):345-360.

Valenzuela G, L; Vásquez M, R; Rojas G, R; Villalba V, MI; Phillips, O; López G, G; Chama M,
V; Monteagudo M, A; Bellota T, D; Huillca A, Y; Pallqui C, NC. 2015. Línea base para el monitoreo de la vegetación en la Reserva Comunal El Sira (RCS). Arnaldoa 22(1):243-268.

Violle, C; Navas, ML; Vile, D; Kazakou, E; Fortunell, C, Hummel, I; Garner, E. 2007. Let the concept of trait be functional. Oikos 116:882892. 


\section{Anexos}

Anexo 1. Descripción de las características biofísicas del área de estudio según las zonas de vida.

\begin{tabular}{|c|c|c|c|}
\hline & 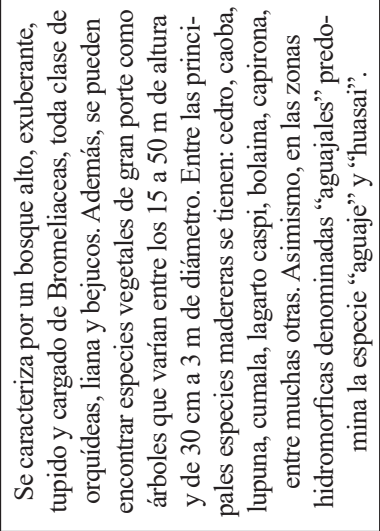 & 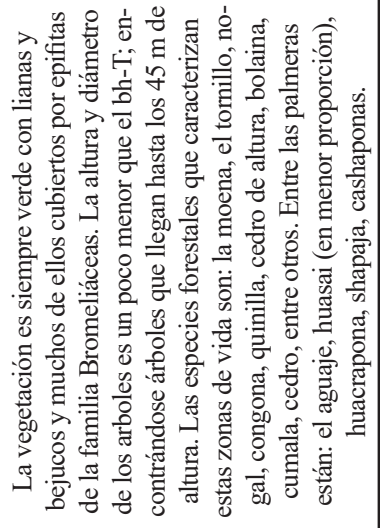 & 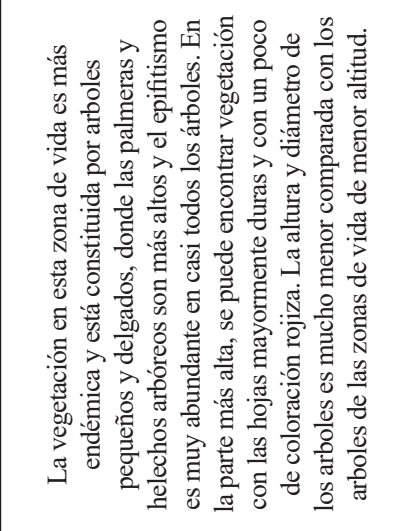 \\
\hline 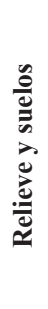 & 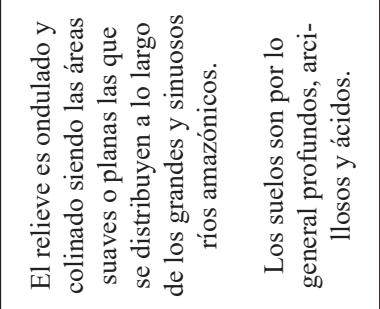 & 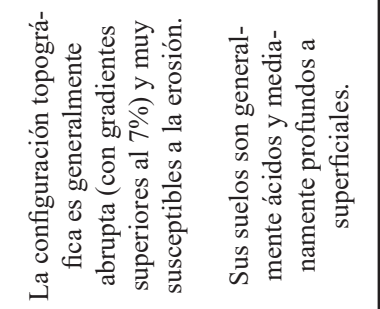 & 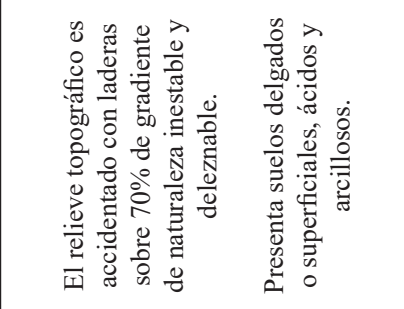 \\
\hline & 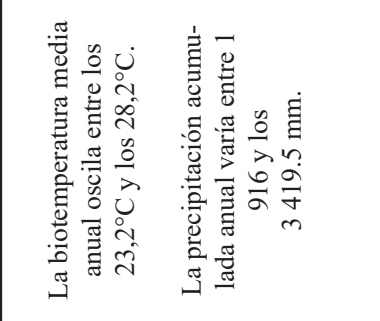 & 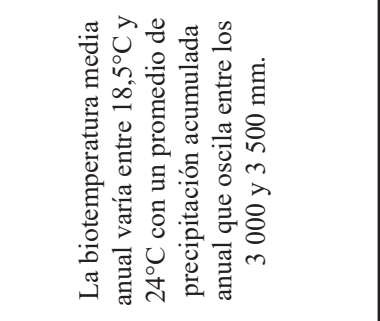 & 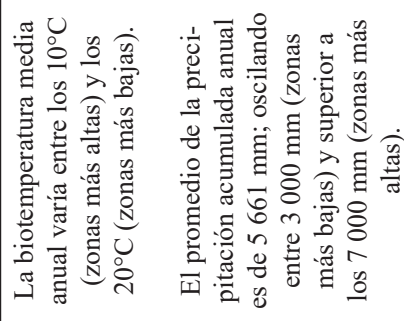 \\
\hline 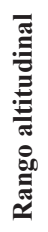 & 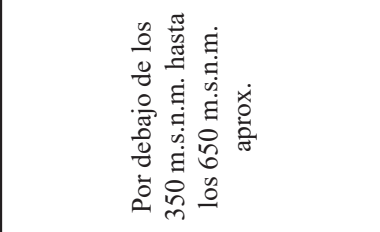 & 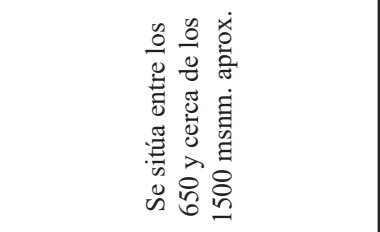 & 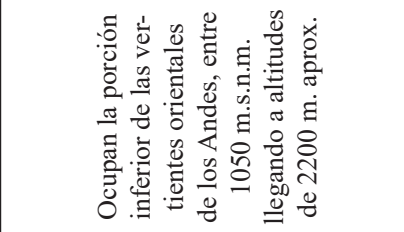 \\
\hline 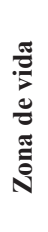 & 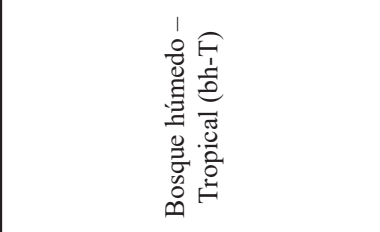 & 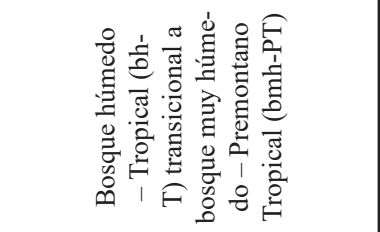 & 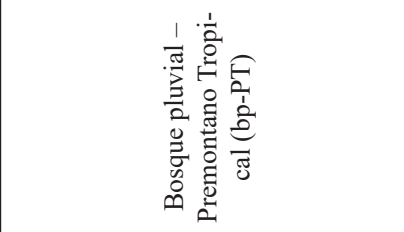 \\
\hline
\end{tabular}

